\title{
Probing young massive clusters with laser guide star adaptive optics
}

\author{
Nate McCrady
}

Received: 10 January 2008 / Accepted: 12 March 2008 / Published online: 24 September 2009

(C) The Author(s) 2009. This article is published with open access at Springerlink.com

\begin{abstract}
We use laser guide star adaptive optics (LGS/AO) on the $10 \mathrm{~m}$ Keck II telescope to obtain high spatial resolution images of young massive clusters (YMCs) in NGC1569 and M82. These data probe YMC structure and the relation of the YMCs to the ambient field star population. The higher resolution of Keck LGS/AO relative to Hubble Space Telescope/NICMOS in the near-infrared enables us to examine whether YMCs are monolithic or hierarchical assemblies. The new integral-field spectrometer OSIRIS operating behind LGS/AO can trace the distribution of massive evolved stars within a cluster and reveal the nature of mass segregation.
\end{abstract}

Keywords Galaxies: star clusters - Methods: observational

\section{Adaptive optics observations}

Most stars form in clusters or loose associations (Evans 1999; Lada 1999). Over time, star clusters are subject to various internal and external mechanisms that tend to disrupt and disperse the clusters. Stars lost by the clusters enter the field population of the galaxy. Understanding cluster evolution, specifically their dissolution, is therefore key to understanding the origin of stellar populations within galaxies (Kroupa 2002).

The degree to which a cluster remains a bound object or contributes stars to the general disk population of a galaxy

\section{N. McCrady $(\varangle)$}

Division of Astronomy \& Astrophysics, UCLA, Los Angeles,

USA

e-mail: nate@astro.ucla.edu depends in large part upon the cluster's internal structure. Investigation of extragalactic YMCs requires the highest available angular resolution, as the typical sizes of clusters are $1-5 \mathrm{pc}$, and ground-based seeing of $1^{\prime \prime}$ corresponds to a spatial extent of nearly $20 \mathrm{pc}$ at a distance of $4 \mathrm{Mpc}$.

Our adaptive optics work is motivated by three outstanding questions regarding the structure and evolution of young massive clusters (YMCs):

1. Are YMCs monolithic objects or assemblies of substructures?

2. When YMCs evolve over time, they disperse. What is the observational relationship of clusters to ambient field stars?

3. What constraints can we place on the nature of mass segregation in YMCs?

Each of these questions requires high spatial resolution observations. Subarcsecond resolution is required to study clusters on pc scales in even the nearest star-forming galaxies. So what resources are available for high angular resolution imaging? Prior to the loss of the Advanced Camera for Surveys (ACS) on the Hubble Space Telescope (HST), its High Resolution Camera could deliver $\sim 60$ milliarcsecond (mas) resolution in the optical. This is less than a pc at the distance of nearby dwarf galaxy NGC1569. In the near-infrared (near-IR), HST still offers NICMOS, where NIC2 has resolution of $\sim 170$ mas in the $H$ band-better than ground-based seeing but still several pc at the distance of NGC1569 or M82. The laser guide star adaptive optics (LGSAO) system at the Keck Observatory uses a 10-14 W $\mathrm{Na} D$ laser to place an artificial guide star of $V \sim 10 \mathrm{mag}$ almost anywhere in the sky. With the 2007 upgrade of the wavefront sensor, the LGSAO system can deliver correction at up to $2 \mathrm{kHz}$ and Strehl ratios in excess of $50 \%$ at $2.2 \mu \mathrm{m}$. 
Figure 1 compares the $H$-band resolution of Keck LGSAO and HST/NICMOS images of the YMC NGC 1569-B. Diffraction-limited AO images take full advantage of Keck's larger primary mirror to deliver four times better angular resolution than HST in the near-IR. Archival $\mathrm{ACS} / \mathrm{HRC}$ images can match the AO image resolution in the optical, but in dusty starbursts like M82, longer-wavelength near-IR LGSAO observations offer a significant advantage.
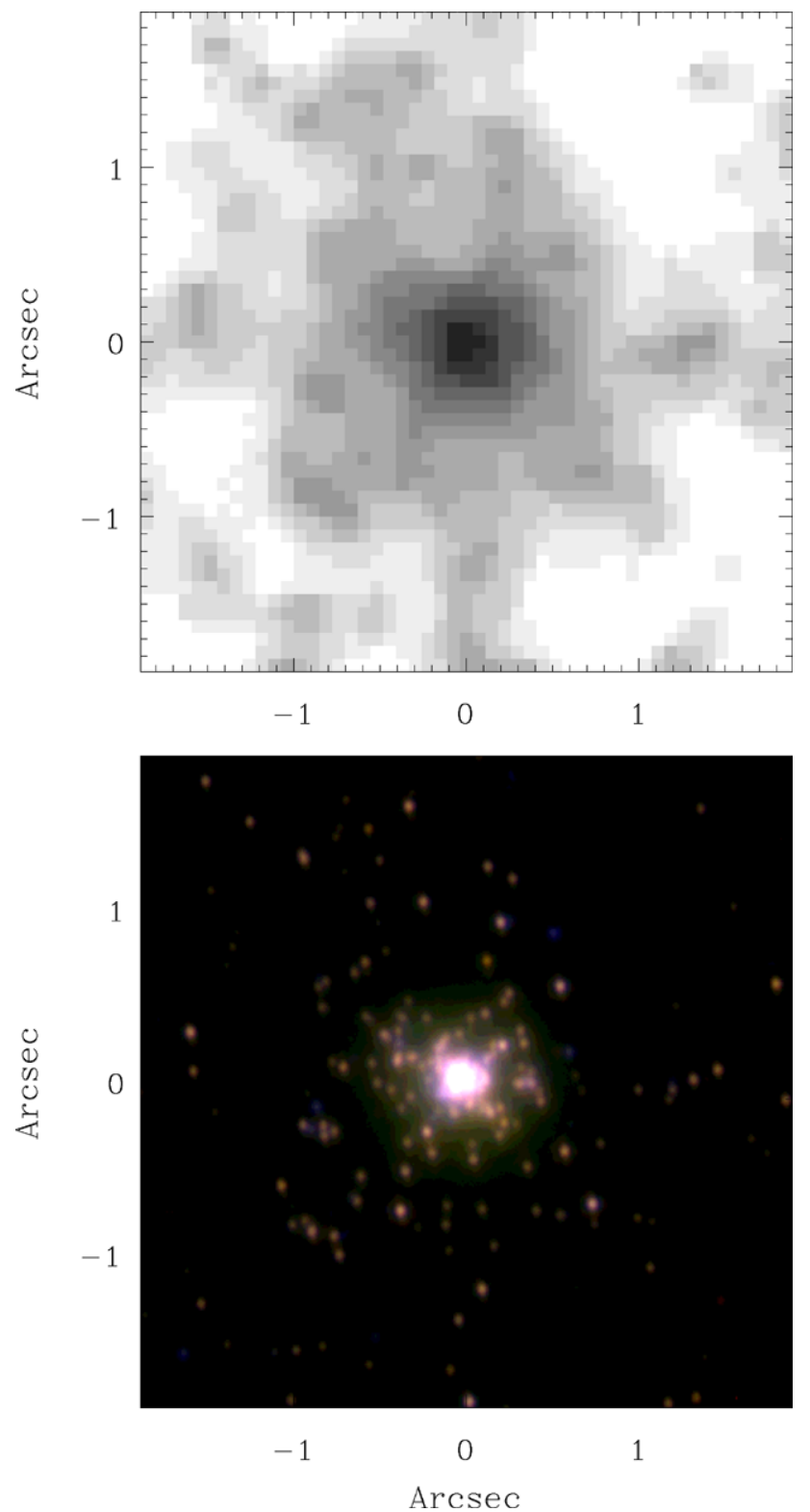

Fig. 1 Top: HST/NICMOS (NIC2) F160W image of cluster NGC1569-B. Bottom: IHK mosaic of the same field, with Keck LGSAO $H$ and $K^{\prime}$ images and ACS/HRC F814W $(I)$ image. With four times the angular resolution of $H S T /$ NICMOS, LGSAO images resolve the cluster and reveal substructure in the core. At the distance of $\mathrm{NGC} 1569,1^{\prime \prime} \sim 12 \mathrm{pc}$

\section{Cluster structure}

Super star cluster NGC1569-A is known from spectroscopy to have two velocity components. With LGSAO images, we can resolve the double cluster on subarcsecond scales ( $\sim 0.6 \mathrm{pc}$ ). A bright source dominates the light of the central region of the cluster. By fitting a King profile to this source and subtracting it from the image, we can reveal a small collection of fainter resolved sources. This is the first resolved imaging of this double cluster.

M82 is a nearby $(3.6 \mathrm{Mpc})$ dusty, highly inclined starburst galaxy, and thus a favorite target for infrared astronomers. ACS/HRC BVI images of the nuclear region show prominent foreground dust lanes along the major axis of the galaxy. The optically bright cluster M82-F lies just to the side of one broad lane, and the very red cluster M82-L lies in the midst of the strong extinction. In LGSAO $H$ and $K$-band images, M82-L is much brighter than M82-F. Several other clusters, notably diffuse M82-a and compact M82-y, are readily evident in the near-IR but fully extinguished in $B V I$ images.

M82-a appears as a bright central region surrounded by a radially diminishing distribution of point sources (Fig. 2). M82-L shows a similar pattern, though the cluster is brighter and more centrally concentrated than M82-a. We fit a King profile to the radial profile of the inner 120 mas core of M82-L, and find that about a third of the integrated light in the cluster lies in excess of the fit at mid to large radii. Repeating the exercise in M82-a shows that nearly two-thirds of that cluster's light is in excess of the best King profile

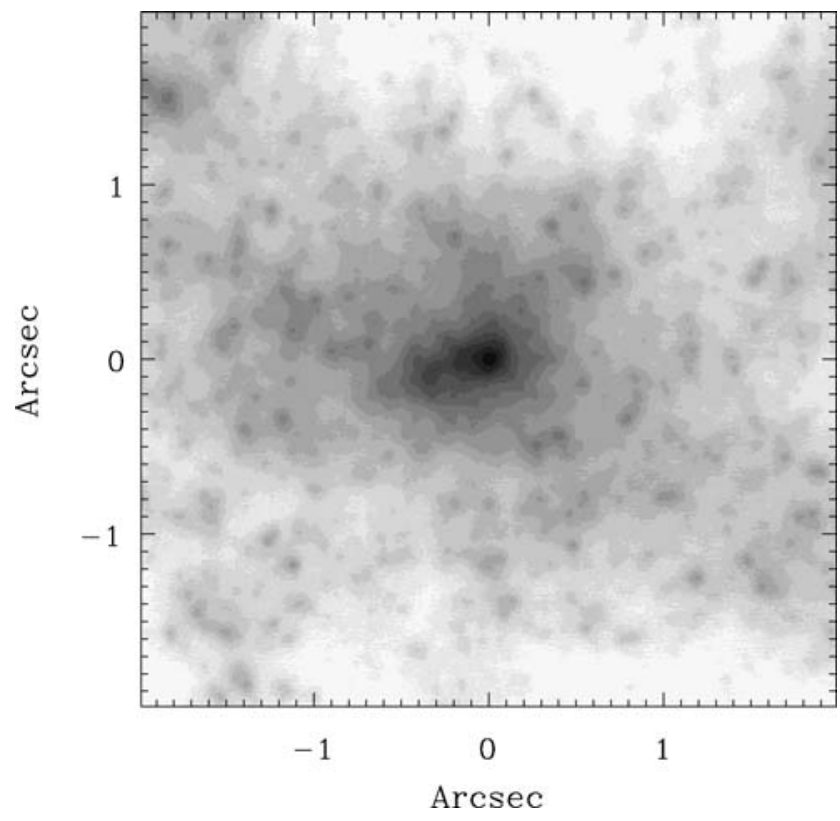

Fig. 2 Keck/LGSAO $H$-band image of M82-a. The compact center of the cluster is surrounded by a swarm of point sources. High extinction renders M82-a invisible in optical images 


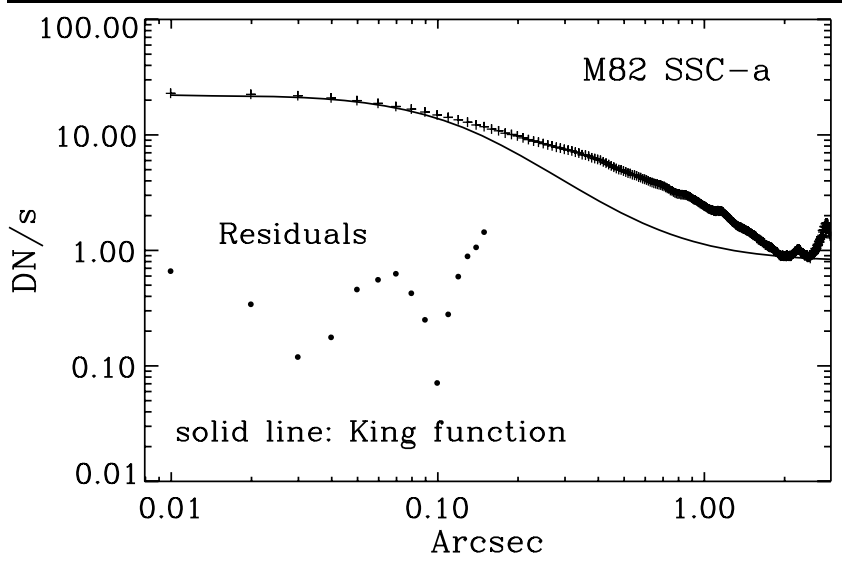

Fig. 3 Fit of a King model to the light profile of the inner 120 mas of M82-a. The plot indicates that a significant proportion of the light of the cluster is in excess of the best King profile at large radii, suggesting that the cluster may be losing stars to the field

fit (Fig. 3). These data suggest that the clusters are losing stars to the field even at their young ages of 10-50 Myr. It is important to emphasize that a two-dimensional fit to the inner 120 mas of the cluster encompasses $\sim 30$ resolution elements! The images clearly resolve the inner regions of the clusters.

\section{Relation of a cluster to ambient field stars}

Galaxies are dangerous places for YMCs. Various external mechanisms, including tidal evaporation, disk shocking and dynamical friction, operate over time to destroy the clusters. Low-mass clusters are particularly vulnerable to these processes (Ashman and Zepf 1998), and are preferentially destroyed (Fall and Zhang 2001). The small galactocentric radii of many starburst clusters tends to make them very prone to disruption by dynamical friction. In addition, there is growing evidence that many clusters dissolve within the first 10 Myr from birth as a result of loss of residual natal gas (Goodwin and Bastian 2006). YMCs, with ages of around $10 \mathrm{Myr}$, thus offer the opportunity to study the dissolution process as it is occurring.

We are using AO imaging to investigate the dissolution of nearby YMCs by measuring their light profiles and plotting color-magnitude diagrams of stars in their vicinity. The high angular resolution of AO imaging enables us to do photometry in crowded fields at the edges of cluster cores and in their extended halos. As noted above, we have detected substantial light in excess of best fit equilibrium profiles in M82 YMCs, and infer that these young clusters are seen in the process of losing stars to the field. This observational result appears to confirm predictions of simulations of star loss during violent relaxation in young clusters.
The high spatial resolution of LGSAO enables us to probe the links between young massive clusters and the surrounding field stars. In $I H K$ color mosaics of the double cluster NGC1569-A, stars of a wide range of colors can be identified out to several half-light radii around the cluster core. Application of our crowded-field AO photometry technique (Sheehy et al. 2006) will enable color-color and color-magnitude studies of the ambient stars and facilitate comparisons to the stars of the cluster.

\section{Mass segregation}

Investigation of mass segregation in YMCs requires spatially resolved spectroscopy to characterize the radial distribution of the most massive stars in the clusters. Newly available integral-field-unit (IFU) spectrometers operating behind AO systems provide the opportunity to obtain spatially resolved spectroscopy at moderate spectral resolution $(\lambda / \delta \lambda \sim 3500)$. In a pilot study conducted in July 2007 , we used the OSIRIS IFU at the Keck II telescope to observe a YMC in NGC6946. This cluster displays a color gradient, with the cluster growing bluer towards larger radii (Larsen et al. 2001). This is may be indicative of mass segregation, wherein the light at the center is dominated by red supergiant stars, the progenitors of which were highmass main-sequence stars. OSIRIS observations reveal the spatial distribution of high-mass red supergiant stars within a cluster via spectral signatures such as $\mathrm{CO}$ absorption bandheads. Application of new IFU technology will enable characterization of the distribution of the most massive, evolved stars of many YMCs within $10 \mathrm{Mpc}$. This will facilitate comparison to nearby (Galactic and LMC) resolved clusters and provide insight into the environmental density dependence of the stellar initial mass function.

Acknowledgements I would like to thank my collaborators James R. Graham at UC Berkeley and William D. Vacca at SOFIA-USRA for their integral contributions to this ongoing project. The author and collaborators wish to recognize and acknowledge the very significant cultural role and reverence that the summit of Mauna Kea has always had within the indigenous Hawaiian community. We are most fortunate to have the opportunity to conduct observations from this mountain. This material is based upon work supported by the National Science Foundation under Grant No. 0502649. Any opinions, findings, and conclusions or recommendations expressed in this material are those of the authors and do not necessarily reflect the views of the National Science Foundation.

Open Access This article is distributed under the terms of the Creative Commons Attribution Noncommercial License which permits any noncommercial use, distribution, and reproduction in any medium, provided the original author(s) and source are credited. 


\section{References}

Ashman, K.M., Zepf, S.E.: Globular Cluster Systems. Cambridge University Press, Cambridge (1998)

Evans, N.J.: Annu. Rev. Astron. Astrophys. 37, 311 (1999)

Fall, S.M., Zhang, Q.: Astrophys. J. 561, 751 (2001)

Goodwin, S.P., Bastian, N.: Mon. Not. R. Astron. Soc. 373, 752 (2006)

Kroupa, P.: Mon. Not. R. Astron. Soc. 330, 707 (2002)
Lada, E.A.: In: Lada, C., Kylafis, N. (eds.) The Origin of Stars and Planetary Systems. NATO Science Series C, vol. 540, p. 441. Kluwer Academic, Dordrecht (1999)

Larsen, S.S., Brodie, J.P., Elmegreen, B.G., Efremov, Y.N., Hodge, P.W., Richtler, T.: Astrophys. J. 556, 801 (2001)

Sheehy, C.D., McCrady, N., Graham, J.R.: Astrophys. J. 647, 1517 (2006) 